\title{
Hilbert-Huang transform in detecting and analyzing the uterine contraction activities
}

\author{
Kemal Aydın', Murat Demirer², Coşkun Bayrak ${ }^{3}$ \\ 'Department Computer Science, North American University, Houston, Texas, United States of America \\ ${ }^{2}$ Department of Computer Engineering, Üsküdar University, İstanbul, Turkey \\ ${ }^{3}$ Department Computer Science, University of Arkansas at Little Rock, Little Rock, Arkansas, United States of America
}

\begin{abstract}
Objective: The diagnosis of labor is currently one of the most difficult problems encountered by obstetrical healthcare providers. A major health problem is the increase in the rate of preterm delivery, which is responsible for $75 \%$ of all deaths in newborns. In addition, preterm delivery is associated with several cognitive and health problems in later life and enormous costs for the health system. A better understanding of myometrial activities could help to reduce preterm deliveries and the costs associated with prematurity in the following years. Therefore, the objective of this study was to determine whether using the Hilbert-Huang transform (HHT) to analyze the uterine contraction data would help us gain a better insight of the myometrial activities of the human uterus during pregnancy.

Material and Methods: Uterine magnetomyographic (MMG) signals were recorded from pregnant patients at gestational ages of 32-38 weeks. The study was approved by the Human Research Advisory Board of the University of Arkansas for Medical Sciences (UAMS) and performed after obtaining written consent from each patient. The recording of transabdominal MMG signals was conducted with the SQUID Array for Reproductive Assessment (SARA, VSM MedTech Inc; Coquitlam, BC, Canada) system, which has 151 primary magnetic sensors allocated approximately $3 \mathrm{~cm}$ apart over an area of $850 \mathrm{~cm}^{2}$. The arrangement of sensors is concave in nature and, in a similar lateral distance, spans the maternal abdomen longitudinally from the symphysis pubis to the uterine fundus. The recording times ranged from 12 to $28 \mathrm{~min}$, and the sampling rate was $250 \mathrm{~Hz}$. The data were down-sampled to $25 \mathrm{~Hz}$ to reduce the computational complexity and post-processed with a bandpass filter $(0.05-1 \mathrm{~Hz})$ because the uterine contraction activity is a band-limited process $(0.05-1 \mathrm{~Hz})$. The recordings of one intrauterine pressure catheter (IUPC) dataset and two mother-perceived contraction datasets were compared with the HHT results, and HHT's potential was explored through the development of a module and a series of experiments. The local energy and the instantaneous frequency derived from the intrinsic mode functions (IMFs) through HHT provide a full energy-frequency-time distribution of the data. Our objective was to determine whether HHT for each channel can help identify and localize contractions in the uterus. Human studies have been reviewed by the appropriate ethics committee and have therefore been performed in accordance with the ethical standards described in an appropriate version of the 1975 Declaration of Helsinki, as revised in 2000.

Results: After comparing the IUPC and other mother-perceived contraction (STIM) datasets with HHT results, we were able to visually detect contraction locations in the HHT-processed uterine signals. For verification and validation purposes, when we further analyzed the delay time between two signals, the mechanical activity (i.e., IUPC) following the electrical activity (i.e., magnetic signal) was observed. In conclusion, our experimentations using the method introduced here revealed that there is a 75\% correlation between the results obtained by HHT and IUPC data. Conclusion: This study compared uterine contractions and changes in the intrauterine pressure with results obtained by HHT. In addition, using IUPC data as a validation guide, we showed that the HHT approach can be used for noise removal. There is a need for time-saving and non-subjective automatic contraction detection in the field of prenatal examination. (J Turk Ger Gynecol Assoc 2015; 16: 195-202)
\end{abstract}

Keywords: Uterine contraction, myometrium, magnetomyographic activity, Hilbert-Huang transform, empirical mode decomposition, contraction analysis

Received: 12 March, 2015

Accepted: 23 August, 2015

Available Online Date: 02 November, 2015

\section{Introduction}

The purpose of this work was to identify uterine contractions using the Hilbert-Huang transform (HHT) to gain a better insight of the myometrial activities of the human uterus during pregnancy. The myometrium is the muscle layer in the human uterus and consists of small smooth muscle cells. The coordinated activity of these muscles is responsible for the generation of uterine mechanical contractions, which leads to the delivery of the fetus. The diagnosis of labor is currently one of the most difficult problems encountered by obstetrical healthcare providers. Prediction of labor is important in both normal and complicated pregnancies. A major health problem is the increase in the number of preterm deliveries, which are responsible for $75 \%$ of all deaths in newborns $(1,2)$. In addition, preterm delivery is associated with several cognitive and health problems in later life and enormous costs for the health system (3). A better understanding of myometrial 
activities could help reduce preterm deliveries and the costs of prematurity in the following years. Apart from recording the changes in the cervical state, the progress of labor is monitored by measuring the rate, duration, and amplitude of uterine contraction using a tocodynamometer (TOCO) or the measurement of intrauterine pressure using an intrauterine pressure catheter (IUPC). Because of its simplicity and almost risk-free nature for the mother and fetus, the TOCO technique is widely used by physicians. However, its susceptibility to maternal motion artifacts is known to be the major drawback. Compared with IUPC, which is an invasive procedure that requires the rupture of the amniotic membranes, TOCO provides less reliable results. Because of the poor predictive power of TOCO and the invasive nature and limited use of IUPC (4), neither technique has been beneficial in the prediction of preterm labor or the diagnosis of true labor at term. If physicians were able to more objectively differentiate between true and false labor, unnecessary visitations to the hospital and the associated treatment could be avoided.

The SQUID Array for Reproductive Assessment (SARA) (4) system is a non-invasive device that is designed to specifically study different aspects of maternal and fetal physiology, particularly in detecting weak biomagnetic fields associated with the electrophysiological activity in the human body (5). The role of SARA (6) can be summarized as the process of capturing the fluctuating magnetic field activities "generated during the polarization of the biological tissue, using a set of sensors (an array of 151 SQUID sensors) covering the complete area of the maternal abdomen starting from the perineum to the top of the uterine fundus." The advantage of using SARA is that it records magnetomyographic (MMG) activity related to the uterine electrophysiological activity, and this information could provide an opportunity to track the parameters that would aid in the prediction of the onset of active labor. This ability would be of great clinical benefit for the management of term patients and particularly for the management of patients at high risk for premature delivery. Myometrial activity patterns may reveal whether a contraction will lead to a delivery. As a novel approach in gaining new insights into uterine data, HHT, which has already been proven to be successful in various non-stationary and non-linear data analysis (6-10), was examined. HHT can be used to better explain its application in the identification of contraction patterns and data analysis properties. Some of these properties include decomposition and expansion of the data into components that are called intrinsic mode functions (IMF) (11), which reveal interesting information about the original data, localizing events in the time-frequency domain using temporal frequency energy distribution, and noise removal.

\section{Material and Methods}

\section{Data recording}

Uterine MMG signals were recorded from pregnant patients at gestational ages of 32-38 weeks. The study was approved by the University of Arkansas for Medical Sciences (UAMS) Human Research Advisory Board and performed after obtaining written consent from each patient. Transabdominal MMG signals were recorded with the SARA (VSM MedTech Inc., Cocuitlam, BC, Canada) system with an array of magnetic sensors [i.e., 151 primary magnetic sensors spaced approximately $3 \mathrm{~cm}$ apart over an area of $\left.850 \mathrm{~cm}^{2}(4)\right]$. The sensors are arranged in a concave array that spans the maternal abdomen longitudinally from the symphysis pubis to the uterine fundus and a similar lateral distance.

Because of the invasive nature of IUPC recording, simultaneous recordings with SARA were performed only on a single patient. Although for all the subjects, maternal perception of the contraction was also recorded simultaneously with SARA measurements. Therefore, during the recording process, the subjects were asked to use their finger in the up-position as a duration indicator of each perceived contraction to inform the operator to mark the beginning and end time points of the contraction in the record.

The recording times ranged from 12 to $28 \mathrm{~min}$, and the sampling rate was $250 \mathrm{~Hz}$. The data were down-sampled to $25 \mathrm{~Hz}$ to reduce the computational complexity and post-processed with a bandpass filter $(0.05-1 \mathrm{~Hz})$ because the uterine contraction activity is a band-limited process $(0.05-1 \mathrm{~Hz})(8)$.

\section{Data analyses}

Empirical mode decomposition (EMD) (12) is the decomposition of the signal into IMFs and is based on the direct extraction of the energy associated with various intrinsic time scales. These time scales are the most important features of complex biological systems such as the uterus. Among the decomposed IMFs, we selected the one that has the highest standard deviation (SD). The choice of IMF with the highest SD is based on the fact that the manifestation of a contraction will make a large deviation from the signal's average, thereby making the SD higher. The use of Hilbert transform to drive the local energy and the instantaneous frequency from the IMFs provides a full energyfrequency-time distribution of the data. Such representation is designated as the Hilbert spectrum. They have well-behaved Hilbert transforms, as expressed in IMFs, from which the instantaneous frequencies can be calculated. Thus, any contractionrelated event can be localized based upon time.

Decomposition can also be viewed as an expansion of the data in terms of the IMFs. Subsequently, these IMFs based on and derived from the data can serve as the basis of the non-linear expansion of data. The most interesting property of the method is its adaptive nature to data.

\section{Hilbert-Huang transform (HHT) in uterine contractions}

Our aim was to determine whether HHT of each channel can reveal and localize contractions in the uterus. To explore the potentiality of HHT, we developed a system to conduct a series of experiments. We want to note that HHT is more of an empirical approach rather than a theoretical method. It has been successfully applied in many areas such as non-linear ocean wave evolution data; earthquake signals and structure responses; bridge and structural health monitoring; biomedical signals such as blood pressure fluctuations; long-term environmental data, including global temperature variations, 
Antarctic ice extents records, solar irradiance variance; hydromachinery design; and machine vibration. The key feature of HHT is EMD, which provides a unique basis called IMFs for the underlying hidden parts of the data that are derived from data and adaptive to the data.

\section{Empirical mode decomposition (EMD) method}

Two conditions need to be satisfied for any IMF. The first condition refers to the fact where the number of extrema and zero crossings are either equal or differ at the most by one. The second condition refers to the mean value of the envelope defined by the local minima and local maxima being zero at any point. According to these two conditions, the sifting process is applied. In the sifting process, the cubic splines from the local maxima and local minima points of uterine contraction data are calculated. These cubic splines are called envelopes. Then, the average is calculated from the envelopes using cubic spline approximations, and this average is subtracted from the signal. The process is repeated until the resulting mean satisfies the two aforementioned properties of IMF. More details on the use of EMD can be found in (9).

The HHT methodology is described as follows: Let $\mathrm{X}(\mathrm{t})$ be the MMG signal, $\operatorname{IMF}_{\mathrm{i}}(\mathrm{t})$ be the $\mathrm{i}^{\text {th }} \mathrm{IMF}$ function, and $\mathrm{R}(\mathrm{t})$ be the residue of the signal. Then, the original signal $\mathrm{X}(\mathrm{t})$ can be written as follows:

$X(t)=\sum_{i=1}^{n} I M F_{i}(t)+R(t)$

The sifting process is terminated if the difference between the successive IMFs is less than a prefixed tolerance defined as follows:

$$
S D=\sum_{t=1}^{T}\left[\frac{\left(I M F_{(k)}(t)-I M F_{(k-1)}(t)\right)^{2}}{I M F_{(k-1)}(t)}\right]
$$

SD can have a value between 0.2 and 0.3 according to (9). In all of our calculations, we set SD to 0.2 for a more sensitive difference between siftings.

The Hilbert transform is applied to IMFs to obtain the local energy of the contraction. However, applying the Hilbert transform to a non-stationary signal may result in two cases: 1) frequencies that are outside the Fourier spectrum or 2) frequencies that go outside the band limit for a band-limited signal. To prevent these two situations, the Hilbert transform should be applied to narrow band data (10). Fortunately, IMFs are narrow band signals, and they behave well with the Hilbert transform.

The Hilbert transform, $\mathrm{Y}(\mathrm{t})$, of a given signal, $\mathrm{S}(\mathrm{t})$, is as follows:

$Y(t)=\frac{1}{\pi} P V \int \frac{S\left(t^{\prime}\right)}{t-t^{\prime}} d t^{\prime}$, where PV denotes the Cauchy principal value. With this definition, $\mathrm{S}(\mathrm{t})$ and $\mathrm{Y}(\mathrm{t})$ can be used to form the analytical signal $\mathrm{Z}(\mathrm{t})$, defined as follows:

$$
Z(t)=S(t)+i Y(t)=a(t) e^{i \theta(t)}
$$

where time-dependent amplitude $\mathrm{a}(\mathrm{t})$, phase $\theta(\mathrm{t})$, and the instantaneous frequency $\omega(\mathrm{t})$ are defined as follows:

$$
\begin{aligned}
& a(t)=\sqrt{S^{2}(t)+Y^{2}(t)} \\
& \theta(t)=\arctan \left(\frac{Y(t)}{S(t)}\right) \\
& \omega(t)=\frac{d \theta(t)}{d t}
\end{aligned}
$$

$\mathrm{S}(\mathrm{t})$ can be calculated from IMF components using the Hilbert transform, as a generalized expansion of the Fourier transform with the time variable amplitudes and frequencies:

$S(t)=\operatorname{Re} \sum^{n} a_{k}(t) e^{j \int \omega_{k}(t) d t}$

\section{Results}

We applied HHT to the uterine contraction data obtained from 12 patients. In 11 datasets, there was no quantitative estimate of the performance of HHT because the maternal perception of contraction is a subjective measure. However, in all of the 11 cases, there was a good (qualitative) agreement between the two entities (maternal perceived contractions and the ones identified by HHT). We have recorded the maternal perception of the contractions for all the datasets and compared the instances at which the mothers perceived the contractions with the instances of the ones identified by the HHT approach. In most of the recordings, HHT also identified the low-amplitude contractions, which mothers could not perceive and report as contractions. Because there was a good degree of correlation between the events perceived by mothers (the high-amplitude contractions) and those identified by HHT, any quantification of these two entities would make the approach over-appealing; hence, we have not quantified the same.

We were able to visually detect contraction locations in the HHT-processed uterine signals. For verification and validation purposes, we compared our findings with the IUPC data of one patient who had both MMG and IUPC recordings. For this patient, recordings in each sensor lasted for $720 \mathrm{~s}$ (at a sampling frequency of $25 \mathrm{~Hz}$, resulting in 18000 data points). To facilitate the comparison of the result with IUPC data (Figure 1), we added the results of HHT for all sensors together (Figure 2). As mentioned in the earlier section, IUPC was recorded simultaneously with MMG for this subject. We observed that the mechanical 
(IUPC) activity followed the electrical activity. Apart from this delay, there was a significant correlation between them.

To quantify the correlations between the Hilbert energy and IUPC data, a cross-correlation analysis was performed. The cross-correlation function (CCF) between the Hilbert energy and IUPC data is shown in Figure 3. There was a maximum correlation (coefficient) of 0.774 (square of this gives the $\%$ of correlation) between the two quantities at a time shift of -9.72 s. For a negative shift, the IUPC data was shifted backwards in time. Thus, there was a delay of $9.72 \mathrm{~s}$ between the two signals, indicating that mechanical activity (i.e., IUPC) followed the electrical activity (i.e., magnetic signal).

Because MMG obtained from different sensors are heterogeneous in nature, the resulting Hilbert energy obtained (by adding the HHT results across all the sensors) is noisy. To improve the correlation between the two signals, the results obtained from HHT were smoothed by performing moving average (noncausal/zero-phase filter) with a window width of $10 \mathrm{~s}$. The correlation between the smoothed data and IUPC data is shown in Figure 4. The correlation between the signals increased from 0.774 to 0.8684 , with the delay remaining fairly the same value of approximately $9.72 \mathrm{~s}$ (Figure 4).

Because the correlation analysis of a smoothed data is subjective, we performed the analysis as a function of the width of the smoothing window to see the influence of the window width on the correlation. Naively, one would expect the correlation to increase as a function of window width and reach a maximum value when the optimal window width is reached. Figure 4 shows the correlation analysis as a function of the window width. As anticipated, the correlation increased with the increase in window width and reached a maximum value for an optimal width of $27 \mathrm{~s}$. A further increase in the window width resulted in a decrease of correlation because the smoothing process destroyed relevant signal content in addition to minimizing the noise.

The dependence of delay on the window width is shown in Figure 5. The delay remained fairly constant (stable) at around $-9.72 \mathrm{~s}$ to a window width of $56 \mathrm{~s}$. Although the smoothing process reduced the correlation beyond the width of $27 \mathrm{~s}$ (Figure 5), the delay was quite robust to window width.

\section{Noise separation with HHT}

The reliability measurement was conducted in two different forms: a) the addition of a Gaussian noise (white noise) and b) the addition of a filtered noise. With Gaussian Noise, we show the reliability rate of HHT in the process of removing noise from the signal. Therefore, we added white noise to the signal and then used HHT to retrieve the original signal (Figure 6). Here, the first plot shows the original signal contaminated with a white noise. The second plot shows that the noise was removed fairly well. The third plot is the corresponding IUPC data. To test the strength of HHT, we increased the noise level and conducted additional experiments to show the noise removal from the mixture for obtaining the original signal. The small deviation encountered in the resulting HHT signal is insignificant because the noise is quite large and it still corresponds to the IUPC (80\%). We further increased the noise level and conducted

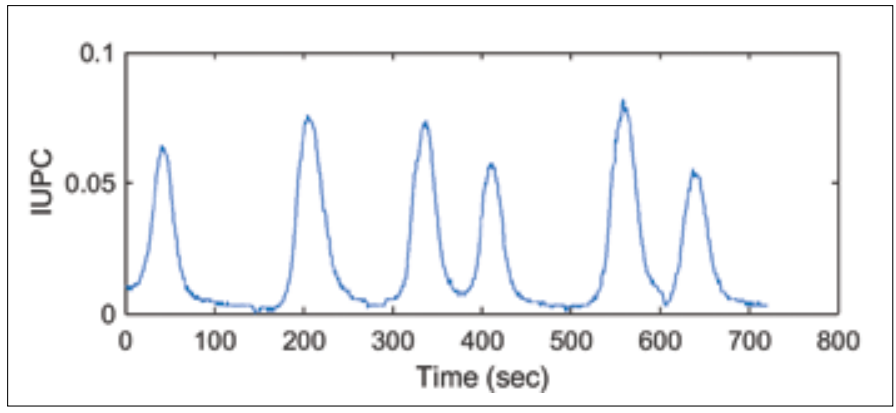

Figure 1. IUPC data

IUPC: intrauterine pressure catheter

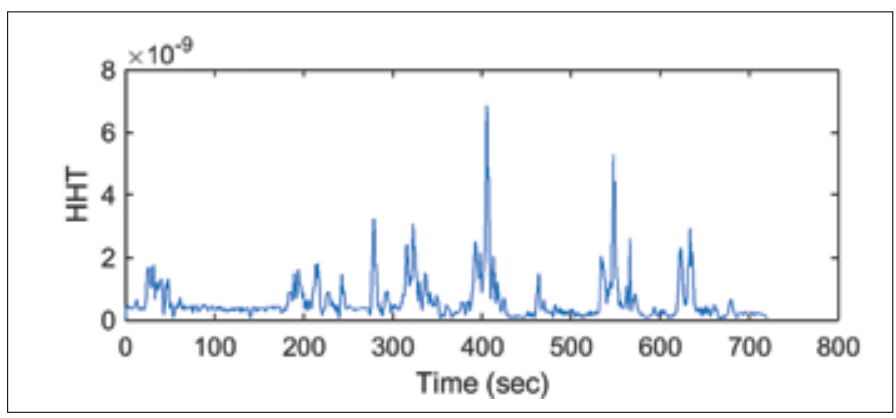

Figure 2. HHT (sum of all channels)

HHT: Hilbert-Huang transform

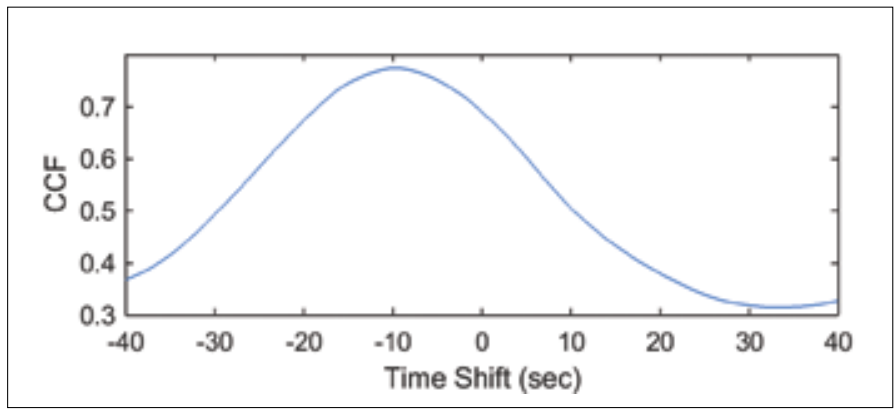

Figure 3. Cross-correlation between the HHT and IUPC data

HHT: Hilbert-Huang transform; IUPC: intrauterine pressure catheter; CCF: cross correlation function

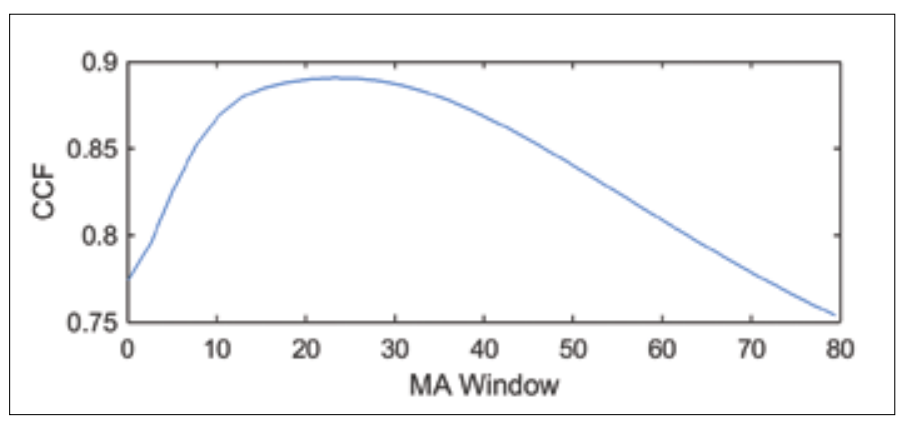

Figure 4. Cross-correlation Function at time shift corresponding to the delay vs. moving average window width

CCF: cross correlation function; MA Window: moving average window

the test again and, as expected, some small contractions were lost, but were still capable of decomposing the dominant contractions from a signal which resembled nothing but a noise. Aiming to take the reliability testing to the next level, we made 


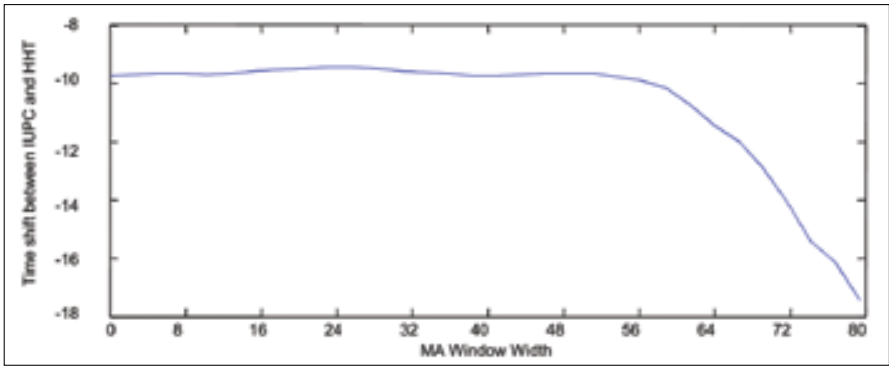

Figure 5. Time shift between IUPC and HHT vs. moving average window length

IUPC: intrauterine pressure catheter; HHT: Hilbert-Huang transform

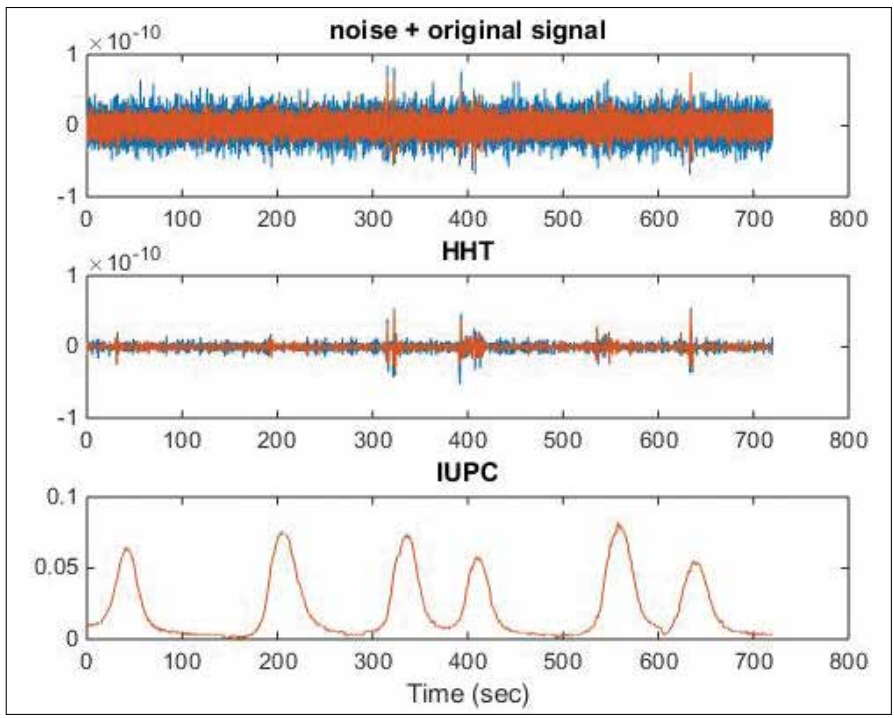

Figure 6. Demonstration of the reliability of HHT using white noise HHT: Hilbert-Huang transform; IUPC: intrauterine pressure catheter

Top panel represents the magnetomyographic (MMG) signal from one of the The SQUID Array for Reproductive Assessment (SARA) sensors with white noises (signalto-noise ratio $(\mathrm{SNR})=0.4501 \mathrm{~dB}, \mathrm{SNR}=-5.5705 \mathrm{~dB}, \mathrm{SNR}=-9.0923 \mathrm{~dB}$ ) added to it. Middle panel represents the Hilbert amplitude of the signal shown in the top panel. Bottom panel represents the intrauterine pressure recorded by intrauterine pressure catheter (IUPC).
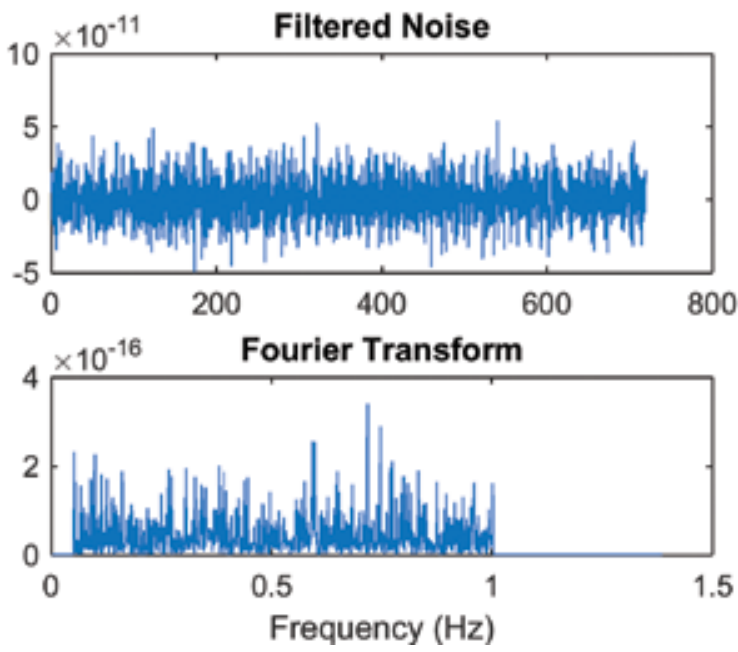

Figure 7. The filtered noise and its Fourier amplitude

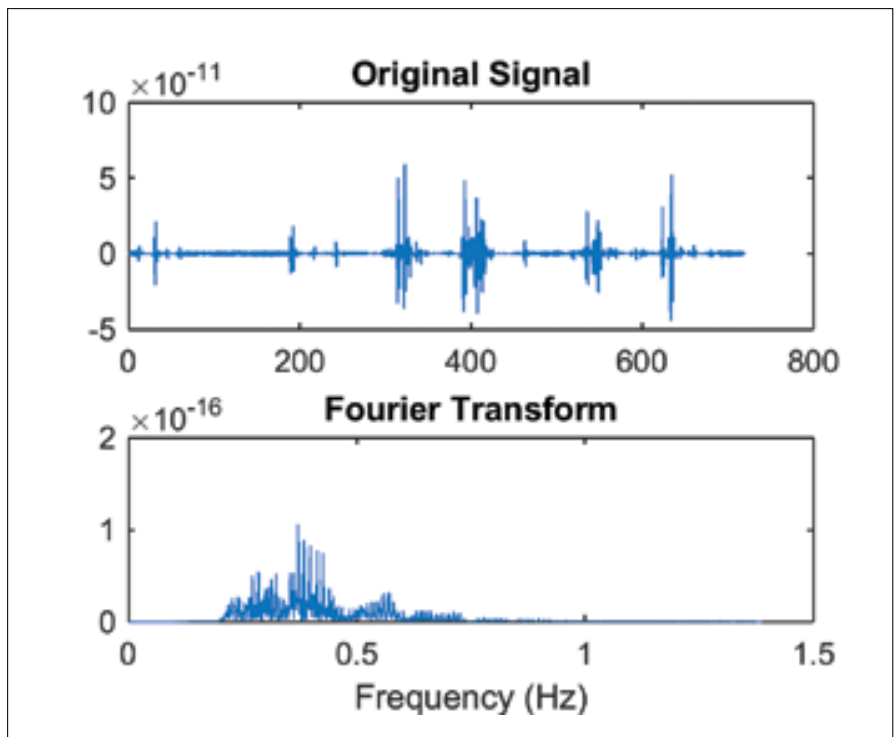

Figure 8. Original signal and its frequency content

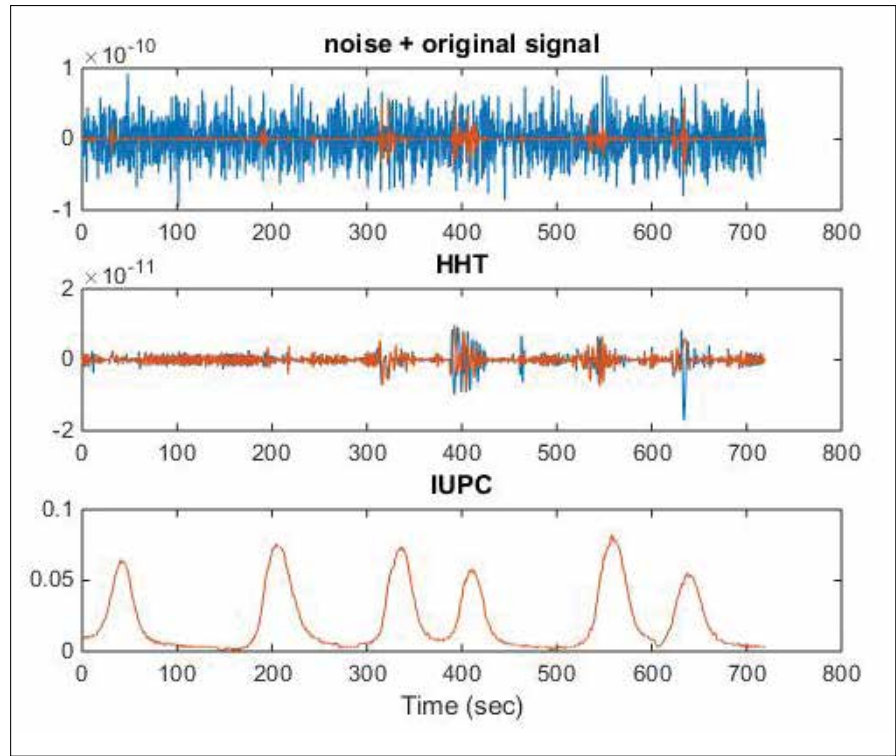

Figure 9. Demonstration of the reliability of HHT using filtered white noise

HHT: Hilbert-Huang transform; IUPC: intrauterine pressure catheter

The quantities plotted are the same as in Figure 6, but with different signal-to-noise ratios (SNRs), i.e., SNR=0.4501 dB, SNR=-5.5705 dB, SNR $=-9.0923 \mathrm{~dB}, \mathrm{SNR}=-11.5911 \mathrm{~dB}$.

the frequency content of the noise, similar to the original signal in the following section.

In the previous section, the use of HHT revealed the success of white noise removal from the signal. However, the noise used has a different frequency content compared with the original signal which makes separation easier. More realistic results can only be obtained by employing filtered noise. Therefore, the white noise was filtered between 0.05 and $1 \mathrm{~Hz}$ to complement the original signal's frequency range (also $0.05-1 \mathrm{~Hz}$ ). Figure 7 depicts the graph of the filtered noise $(0.05-1 \mathrm{~Hz})$ and its frequency content, while Figure 8 depicts the graph of the original signal and its frequency content. 

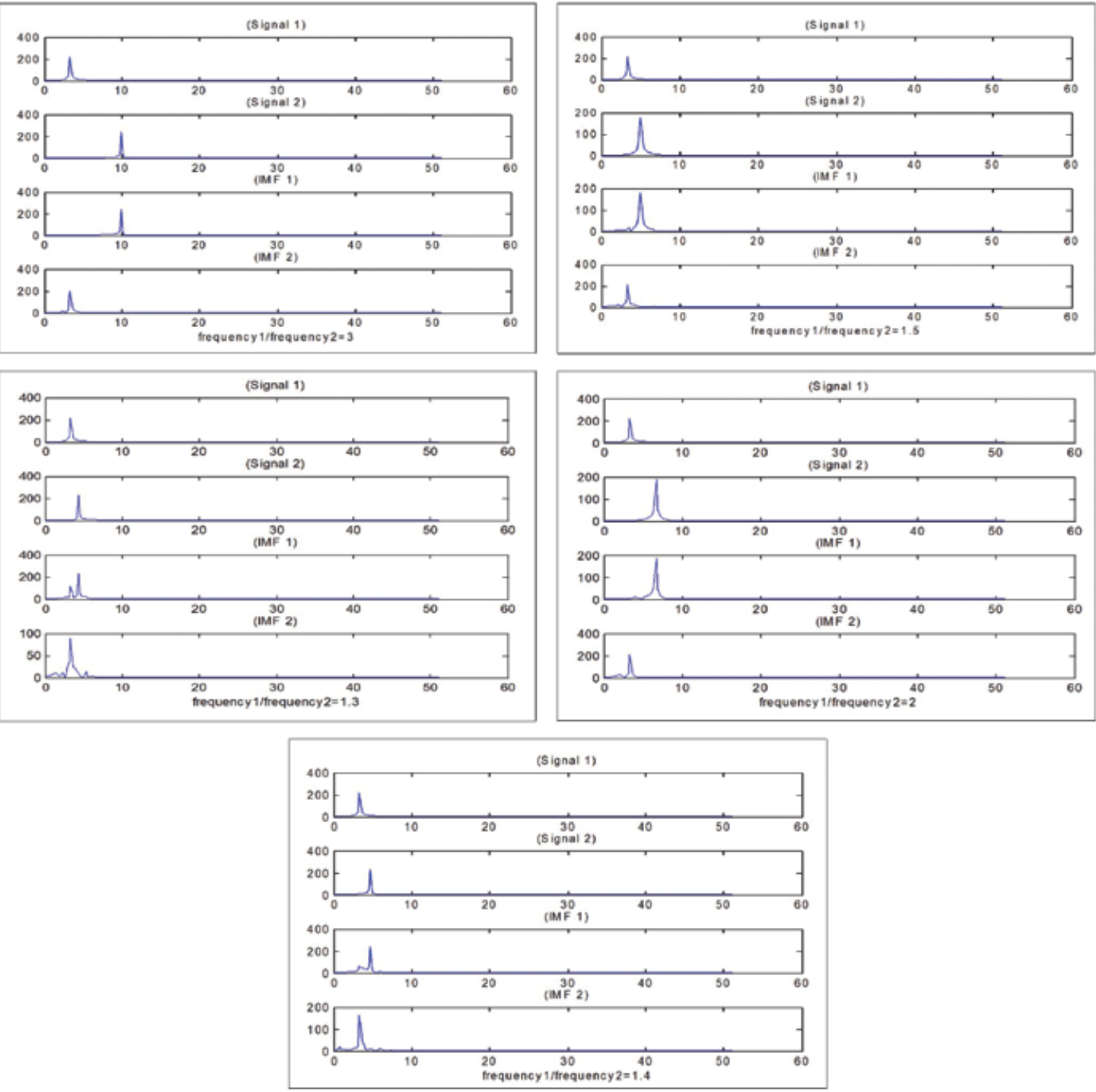

Figure 10. Frequency content of the signals

IMF: intrinsic mode function

The first two plots show the frequency content of each signal, and the second two plots show the frequency of the decomposed IMFs.

Figure 9 show the decomposition of the original signal from a noise-contaminated signal. Here, both the signal and the noise have the same frequency content. Therefore, the separation is an extremely difficult task. The success in separating noise decreased when the noise had the same frequency range as the original signal. Therefore, what should the minimum frequency ratio between two signals be to reliably separate those signals? To provide an answer, we used synthetic data to investigate this issue. Our experiments showed that if the ratio decreases below 1.5, then separating two signals becomes problematic.

Verification and validation of HHT in the separation of signals For each figure in Figure 10, the first and second plots show the frequency contents of each signal. Those signals were mixed together, and HHT was then used to decompose them back from this mixture. The third and fourth plots of each figure show 
the frequency content of the decomposed IMFs. Notably, there are no problems in decomposing two signals if they have a frequency ratio of 1.5 or higher. In other words, if the ratio is greater than or equal to 1.5, decomposed IMFs give the exact frequency contents of the original signals.

As seen in Figure 10, the EMD algorithm is able to separate signals if their frequency ratio is higher than 1.5. When the ratio decreases below this point, the resulting IMFs decomposed from the original signal, start to include data from both signals.

\section{Comparison of HHT with mother perception}

Mother-perceived contractions were recorded in the STIM channel. We compared our findings with STIM data for the additional reliability test of HHT approach. If we encountered a contraction which corresponds to STIM data, we counted it as a true positive, but if we encountered a contraction which did not correspond to STIM data, we did not count it as false positive because we do not expect the mother to feel every contraction. There may be a different threshold for different mothers where they can feel the contraction. Data used in this work were gathered from the records of two patients.

Figure 11 depicts HHT with mother's perception. When a mother felt a contraction, she pushed the STIM button when she thinks it has ended, and she pushed the button again with the assumption a new contraction started. In the figure, the mother perceived two contractions between 90-210 s and 610-695 s. Our HHT plot overlaps with those results; in addition, it shows another contraction at $310 \mathrm{~s}$ and $410 \mathrm{~s}$, which is not recorded by the mother. Figure 12 shows another contraction perceived by the mother between 410 and $550 \mathrm{~s}$. However, the mother did not record the contraction between 600 and $660 \mathrm{~s}$. This may be due to the pressure not reaching the threshold level because HHT shows an increase followed by a large decrease in the electrical activity around $610 \mathrm{~s}$ and then the same activity is repeated around $640 \mathrm{~s}$.

\section{Discussion}

The objective of this study was to determine whether using HHT to analyze the uterine contraction data would help us gain a better insight of the myometrial activities of the human uterus during pregnancy. Based on the results obtained from the experiments, HHT appears to be a promising approach in detecting uterine contractions and shows pressure changes in the womb without any invasive measurement such as IUPC. With appropriate filtering, a 75\% correlation (square of the correlation coefficient, 0.8684 ) was revealed between the HHT and IUPC data, and there exists a delay of $9.72 \mathrm{~s}$ between electrical activity and mechanical activity. In addition to correlation, mother-perceived contractions also support HHT results.

Through the use of the EMD algorithm, noise was removed from the signal, thus discovering if the noise has the same frequency content as the original signal, it becomes harder to remove the noise from the signal. Our experiments showed that for a reliable separation of signal from noise, the ratio of the frequency

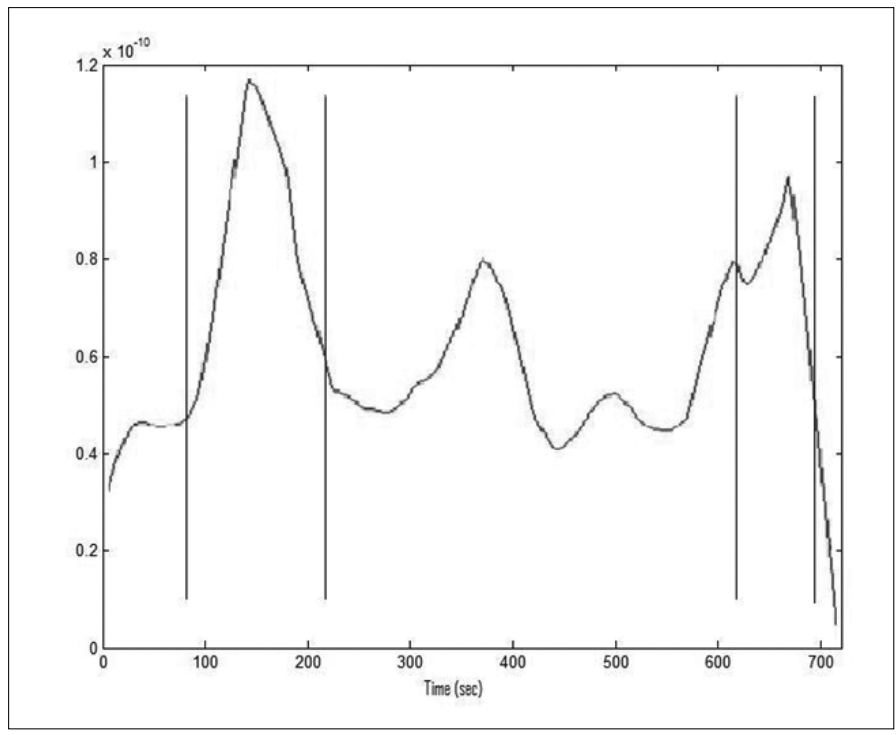

Figure 11. Mother's perception of two contractions



Figure 12. Mother's perception of one contraction

contents should be at least 1.5. There is a need for timely, efficient, optimized, and non-subjective automatic contraction detection approaches in the field of prenatal examination. It is the intension of this study group to revise and refine the exiting contraction detection algorithm to be useful in the near future. With the current system, it is possible to identify the antipodal activities in the cellular community.

\section{Limitations}

Although the SARA system covers the entire pregnant abdomen with 151 sensors recording the magnetic field, at the rate of 250 samples/s and re-sampled with 25 samples/s, generated by the uterine myometrium, the primary frequency band of the uterine contractions are restricted between $0.1 \mathrm{~Hz}$ and $0.2 \mathrm{~Hz}$, which represents underlying contraction activities (13). At this low frequency band rate, we are forced to use a window size of $20 \mathrm{~s}$ for computing the synchronization indices. To avoid the unneces- 
sary distribution of the information collected from the uterine contractions, it is necessary to use small steps, more realistic windows size, and magnetic field data between 0.3 and $1 \mathrm{~Hz}$ for accurate analyses.

Also, the data used in this experimentation is pre-processed, i.e., that the original noise has been removed, and the effectiveness test was conducted using white noise. To test the reliability of the system, a new study is in progress to validate the complimentary findings of the method introduced here.

Ethics Committee Approval: Ethics committee approval was received for this study from the ethics committee of the Institutional Review Board (IRB) (Protocol No \#15-057).

Informed Consent: Written informed consent was obtained from patients who participated in this study.

Peer-review: Externally peer-reviewed.

Author Contributions: Concept - K.A., R.M.D., C.B.; Design - K.A., C.B.; Supervision - C.B.; Resource - K.A., C.B.; Materials - K.A., C.B.; Data Collection and/or Processing - K.A.; Analysis and/or Interpretation - R.M.D., C.B.; Literature Search - K.A., R.M.D., C.B.; Writing - K.A., C.B.; Critical Reviews - R.M.D.

Acknowledgements: This research is partly supported by National Institute of Biomedical Imaging and Bioengineering (NIBIB) of National Institute of Health under the grant number 1R33EB00978-02. We also extend our thanks for Rathinaswamy $B$. Govindan for his valuable comments and feedbacks.

Conflict of Interest: No conflict of interest was declared by the authors.

Financial Disclosure: The authors declared that this study has received no financial support.

\section{References}

1. Report of the U. S. Preventive Services Task Force. Guide to Clinical Preventative Services: An assessment of the effectiveness of 169 interventions. Second Edition. 1989.
2. Brown ER. Long-term consequences of preterm birth. In: Fuchs F, Stubblefield PG, editors. Preterm birth: causes, prevention, and management. New York: McMillan Publishing; 1984. p. 333.

3. Morrison JC, Martin JN Jr, Martin RW, Gookin KS, Wiser WL. Prevention of preterm birth by ambulatory assessment of uterine activity: a randomized study. Am J Obstet Gynecol 1987; 156: 536-43. [CrossRef]

4. Eswaran H, Preissl H, Wilson JD, Murphy P, Lowery CL. Prediction of labor in term and preterm pregnancies using non-invasive magnetomyographic recordings of uterine contraction. Am J Obstet Gynecol 2004; 190: 1598-602; discussion 1602-3. [CrossRef]

5. Radhakrishnan N, Wilson JD, Lowery C, Eswaran H, Murphy P. A fast algorithm for detecting contractions in uterine electromyography. IEEE Eng Med Biol Mag 2000; 19: 89-94. [CrossRef]

6. Eswaran H, Preissl H, Wilson JD, Murphy P, Robinson SE, Lowery CL. First magnetomyographic recordings of the uterine activity with spatial-temporal resolution using 151 channel sensor array. Am J Obstet Gynecol 2002; 187: 145-51. [CrossRef]

7. Norden EH, Shen Z, Long SR. A new view of nonlinear water waves: The Hilbert Spectrum. Ann Rev Fluid Mech 1999; 31: 417-57. [CrossRef]

8. Garcia-Gonzalez MT, Charleston-Villalobos S, Vargas-Garcia C, Gonzalez-Camarena R, Aljama-Corrales T. Characterization of EHG Contractions at Term Labor by Nonlinear Analysis. 35th Annual International Conference of the IEEE EMBS. Osaka, Japan; 2013.

9. Diab MO, Moslem B, Khalil M, Marque C. Classification of Uterine EMG Signals by Using Normalized Wavelet Packet Energy. 16th IEEE Mediterranean Electrotechnical Conference (MELECON); 2012. p. 335-8. [CrossRef]

10. Srhoj-Egekher V, Cifrek M, Medved V. The application of Hilbert-Huang transform in the analysis of muscle fatigue during cyclic dynamic contractions. Med Biol Eng Comput 2011; 49: 659-66. [CrossRef]

11. Magrin-Chagnolleau I, Baraniuk R. Empirical mode decomposition based time-frequency attributes. Proceedings of the SEG Meeting: Houston, Texas; 1999. [CrossRef]

12. Norden EH, Zheng S, Steven RL, Manli CW, Hsing HS, Quanan Z, et al. The empirical mode decomposition and the Hilbert spectrum for nonlinear and non-stationary time series analysis. The Royal Society 1998; 454: 903-95. [CrossRef]

13. Ramon C, Preissl H, Murphy P, Wilson JD, Lowery C, Eswaran H. Synchronization analysis of the uterine magnetic activity during contractions. Biomed Eng Online 2005; 4: 55. [CrossRef] 